\title{
FUT 2 polymorphism and outcome in very-low-birth-weight infants
}

Martin Demmert ${ }^{1}$, Anne Schaper ${ }^{1}$, Julia Pagel' ${ }^{1}$, Corinna Gebauer ${ }^{2}$, Michael Emeis ${ }^{3}$, Friedhelm Heitmann ${ }^{4}$, Angela Kribs ${ }^{5}$, Jens Siegel ${ }^{6}$, Dirk Müller ${ }^{7}$, Annette Keller-Wackerbauer ${ }^{8}$, Hubert Gerleve ${ }^{9}$, Christian Wieg ${ }^{10}$, Egbert Herting', Wolfgang Göpel' and Christoph Härtel'; for the German Neonatal Network

BACKGROUND: To determine whether the secretor gene fucosyltransferase (FUT)2 polymorphism G428A is predictive for adverse outcomes in a large cohort of very-low-birth weight (VLBW) infants.

METHODS: We prospectively enrolled 2,406 VLBW infants from the population-based multicenter cohort of the German Neonatal network cohort (2009-2011). The secretor genotype (rs601338) was assessed from DNA samples extracted from buccal swabs. Primary study outcomes were clinical sepsis, blood-culture confirmed sepsis, intracerebral hemorrhage $(\mathrm{ICH})$, necrotizing enterocolitis (NEC) or focal intestinal perforation requiring surgery, and death.

RESULTS: Based on the assumption of a recessive genetic model, AA individuals had a higher incidence of ICH (AA: 19.0\% vs. GG/AG: $14.9 \%, P=0.04$ ) which was not significant in the additive genetic model (multivariable logistic regression analysis; allele carriers: 365 cases, 1,685 controls; OR: 1.2; 95\% Cl: 0.99-1.4; $P=0.06$ ). Other outcomes were not influenced by FUT2 genotype in either genetic model.

CONCLUSION: This large-scale multicenter study did not confirm previously reported associations between FUT2 genotype and adverse outcomes in preterm infants.

$\mathbf{T}$ he secretor status, i.e., the ability to secrete $\mathrm{ABH}$ histoblood group antigens into body fluids is determined by the enzyme fucosyltransferase 2 which is encoded by the FUT2 gene. About $80 \%$ of the Caucasian populations are secretors (either homozygous SeSe or heterozygous Sese) while the remaining $20 \%$ carry the homozygous $428 \mathrm{G} \rightarrow \mathrm{A}$ nonsense mutation in the FUT2 gene and are nonsecretors (sese). Several in vivo studies have revealed the clinical significance of this polymorphism and the secretor status in terms of susceptibility to infection and association with immunologically mediated diseases (1-6). Thorven et al. (1) were able to demonstrate that secretor-negative individuals are resistant to Norovirus infections possibly due to binding of Norovirus to secreted $\mathrm{ABH}$ histo-blood group antigens as indispensable condition for infection. Another study noted that secretor individuals are overrepresented in a cohort of patients with viral infections of the respiratory tract (2). So far the only investigation involving preterm infants found a strong association between secretor status and severe outcome (i.e., death, necrotizing enterocolitis (NEC), Gram-negative sepsis) in a cohort of 410 premature infants (6).

The aim of this study was to determine possible associations between the FUT2 genotype and short-term as well as longterm outcomes in a large cohort of 2,406 preterm infants with a birth weight $<1,500 \mathrm{~g}$ (very-low-birth weight (VLBW)) from the German Neonatal Network.

\section{RESULTS}

\section{Genotype Frequencies}

In the German Neonatal Network cohort including infants born between 2009-2011, $n=2,566$ infants were enrolled (64.8\% of eligible infants; early death occurred in $21 \%$ of nonenrolled infants). As ethnic differences for FUT 2 genotype distribution have been previously noted, we decided to exclude infants with Asian $(n=48)$, African $(n=75)$, and unknown background $(n=37)$. The genotype frequencies in the remaining cohort of 2,406 infants were in the expected range for Caucasian populations (6-8) and appropriate to allele frequencies, as determined by Hardy-Weinberg equilibrium (FUT2 428 GG: $n=744,30.9 \%$; FUT2 428 GA: $n=1,193,49.6 \%$; FUT2 428 AA: $n=469,19.5 \%)$. The power of this study to detect a difference in mortality rates between AA and AG/GG genotype groups was $99.9885 \%$ at the two-sided $5 \%$ test-level. For power calculations, we used the marginal genotype frequencies of this study, the mortality rates reported by Morrow et al. (6), i.e., 12/95 in the AA genotype group and 13/299 in the AG/ GG genotype group, and the continuity-corrected $\chi^{2}$ test. The power exceeds $99 \%$ even at the two-sided 0.0002 test-level.

'Department of Pediatrics, University at Lübeck, Lübeck, Germany; ${ }^{2}$ Department of Pediatrics, University of Leipzig, Leipzig, Germany; ${ }^{3}$ Department of Neonatology, Vivantes-Klinikum Berlin-Neukölln, Berlin, Germany; ${ }^{4}$ Department of Neonatology, Klinikum Dortmund, Dortmund, Germany; ${ }^{5}$ Department of Pediatrics, University of Cologne, Köln, Germany; ${ }^{6}$ Department of Neonatology, Children's Hospital Auf der Bult, Hanover, Germany; ${ }^{7}$ Department of Neonatology, Klinikum Kassel, Kassel, Germany; ${ }^{8}$ Department of Neonatology, Krankenhaus Barmherzige Brüder St. Hedwig, Regensburg, Germany; ${ }^{9}$ Department of Neonatology, Klinikum Coesfeld, Coesfeld, Germany; ${ }^{10}$ Department of Neonatology, Klinikum Aschaffenburg, Aschaffenburg, Germany. Correspondence: Christoph Härtel (christoph.haertel@uksh.de) 


\section{Clinical Characteristics}

The clinical data of the whole cohort are described in Table 1. We noted differences for causes of preterm delivery stratified to FUT-2 G428A genotype. The incidence of preterm labour was higher in AA individuals compared to GG/AG (39.7 vs. $32.2 \% ; P=0.002$ ), while the incidence for amniotic infection was not different. By contrast, GG/AG individuals were more often delivered due to pre-eclampsia ( 8.1 vs. $3.9 \% ; P=0.002)$ and had a higher likelihood of Caucasian maternal background (88.7 vs. $81.4 \% ; P=0.001$ ) as compared to AA individuals.

\section{Primary Outcomes Investigated in a Recessive Genetic Model}

Based on the assumption of a recessive genetic model (GG/ AG vs. AA), we found no differences according to genotype for clinical sepsis, blood-culture confirmed sepsis, necrotizing enterocolitis or focal intestinal perforation requiring surgery, and death (Table 2). In line with this, the pathogenic spectrum of blood-culture proven sepsis episodes was not different between GG/AG and AA individuals (Table 3). AA individuals were observed to have a nonsignificant association with early-onset sepsis (GG/AG: $1.2 \%$, AA: $2.4 \%, P=0.06$, Fisher's exact test). Interestingly, AA individuals with preterm premature rupture of membranes (PPROM) carried a higher risk to early-onset sepsis as compared to GG/AG individuals with PPROM (5.5 vs. $1.8 \%, P=0.036$ ). We also analyzed the subgroup of VLBW infants $<32 \mathrm{wk}(n=2,076)$ to exclude over-representation of small-for gestational age infants and found no significant risk for early-onset sepsis in that subgroup (GG/AG: $1.4 \%$, AA: $2.3 \% ; P=0.08$, Fisher's exact test). In the recessive genetic model, AA individuals were noted to have a higher incidence of intracerebral hemorrhage (ICH; whole cohort: GG/AG: $14.9 \%$, AA: $19.0 \%, P=0.04$, Fisher's exact test; VLBW infants < 32 wk gestational age: GG/AG: 16.8, AA: $21.6 \%, P=0.03$, Fisher's exact test).

\section{Multivariable Logistic Regression Analysis (Additive Genetic Model)}

Alternatively, we assumed an additive genetic model to test the effect of FUT2 adjusted for well-known clinical risk factors (gestational age per week, birth weight per $100 \mathrm{~g}$ steps, gender, multiple birth, inborn delivery, exposure to antenatal steroids) for the outcomes of interest in a multivariable logistic regression analysis. In the cohort of VLBW infants $<32 \mathrm{wk}$ the FUT2 genotype was noted to have a nonsignificant effect on the development of ICH (365 cases, 1,685 controls; OR: 1.2; 95\% CI: $0.99-1.4 ; P=0.06)$. Likewise, other outcomes including clinical and blood-culture proven sepsis, NEC/focal intestinal perforation requiring surgery and death were not influenced by FUT2 genotype (Table 4). In our cohort, gestational age proved to be the most important predictor for outcome.

Table 1. Clinical characteristics of very-low-birth weight cohort stratified to genotype

\begin{tabular}{|c|c|c|c|c|c|}
\hline Clinical characteristics & All & GG & AG & AA & $P$ value GG/AG vs. AA \\
\hline Number of infants & 2,406 & 744 & 1,193 & 469 & \\
\hline Gestational age (weeks), mean (SD) & $28.8(2.8)$ & $28.7(2.8)$ & $28.8(2.7)$ & $28.7(2.8)$ & \\
\hline Male gender (\%) & 50.0 & 47.7 & 51.8 & 49.0 & 0.64 \\
\hline Multiple birth (\%) & 32.5 & 31.2 & 32.1 & 35.4 & 0.13 \\
\hline \multicolumn{6}{|l|}{ Mode of delivery (\%) } \\
\hline Caesarean section (C/S) & 81.2 & 81.6 & 82.0 & 78.6 & \multirow{2}{*}{0.07} \\
\hline Emergency $\mathrm{C} / \mathrm{S}$ & 9.3 & 9.2 & 9.4 & 9.1 & \\
\hline \multicolumn{6}{|l|}{ Cause of preterm birth (\%) } \\
\hline PPROM & 28.0 & 25.0 & 31.0 & 28.0 & 0.77 \\
\hline Preterm labor & 33.7 & 32.1 & 32.3 & 39.7 & 0.002 \\
\hline Pathological CTG & 19.8 & 18.8 & 20.5 & 19.7 & 0.91 \\
\hline IUGR/path. Doppler & 23.4 & 23.1 & 24.6 & 21.0 & 0.16 \\
\hline Placental abruption & 7.1 & 6.7 & 7.6 & 6.5 & 0.55 \\
\hline Antenatal antibiotics (\%) & 48.2 & 46.3 & 49.3 & 48.1 & 0.99 \\
\hline Antenatal steroids (\%) & 89.6 & 91.6 & 89.8 & 85.9 & 0.004 \\
\hline \multicolumn{6}{|l|}{ Maternal ethnicity (\%) } \\
\hline Caucasian & 88.8 & 89.9 & 87.9 & 81.4 & 0.001 \\
\hline Turkey/Middle East & 11.2 & 10.1 & 12.1 & 18.6 & \\
\hline
\end{tabular}

The cause of preterm birth was set by the attending obstetrician, the documentation of multiple causes was possible; bold text indicates significant associations. C/S, Caesarean section; CTG, cardiotocography; HELLP syndrome (complication of pregnancy including hemolysis, elevated liver enzymes, low platelets); IUGR, intrauterine growth restriction. 
Table 2. Outcomes of very-low-birth weight cohorts stratified to FUT 2 genotype

\begin{tabular}{lrrrrr}
\hline & & & & & $\begin{array}{l}P \text { value } \\
\text { GG/AG }\end{array}$ \\
Outcome measures & All & GG & AG & AA & vs. AA \\
\hline Number of infants & 2,406 & 744 & 1,193 & 469 & \\
Clinical sepsis (\%) & 33.0 & 34.3 & 32.2 & 33.2 & 0.93 \\
B/C proven sepsis & 12.0 & 12.1 & 12.0 & 12.0 & 0.97 \\
Early-onset sepsis (\%) & $\mathbf{1 . 5}$ & $\mathbf{0 . 7}$ & $\mathbf{1 . 6}$ & $\mathbf{2 . 4}$ & $0.08^{\mathrm{a}}$ \\
Late-onset sepsis (\%) & 11.3 & 11.8 & 11.2 & 10.6 & 0.63 \\
Pneumonia (\%) & & & & & 0.12 \\
$\quad$ Early onset & 0.7 & 0.4 & 0.6 & 1.4 & \\
$\quad$ Late onset & 3.1 & 2.3 & 3.3 & 3.7 & \\
Surgery for NEC/focal & 4.5 & 4.2 & 4.6 & 4.4 & 0.93 \\
intestinal perforation (\%) & & & & & \\
Higher stage NEC (\%) & 4.9 & 4.3 & 5.4 & 4.4 & 0.61 \\
ICH (\%) & $\mathbf{1 5 . 7}$ & $\mathbf{1 3 . 9}$ & $\mathbf{1 5 . 6}$ & $\mathbf{1 9 . 0}$ & $\mathbf{0 . 0 4}$ \\
$\quad$ Grade 1 & 6.0 & 5.4 & 6.1 & 6.5 & \\
$\quad$ Grade 2 & 4.0 & 3.5 & 3.5 & 5.9 & \\
$\quad$ Grade 3 & 1.9 & 1.6 & 2.1 & 2.0 & \\
$\quad$ Grade 4 & 3.8 & 3.2 & 3.9 & 4.6 & \\
Periventricular & 3.0 & 2.3 & 3.0 & 4.0 & 0.16 \\
leukomalacia (\%) & & & & & \\
Surgery for VP-shunt (\%) & 1.7 & 0.8 & 2.2 & 2.0 & 0.65 \\
Bronchopulmonary & 14.6 & 13.9 & 15.5 & 13.3 & 0.4 \\
dysplasia (\%) & & & & & \\
Pneumothorax (\%) & 4.9 & 5.3 & 4.7 & 4.8 & 0.93 \\
Death (\%) & 3.6 & 3.9 & 3.3 & 3.9 & 0.69 \\
Nonsurvivors with & 7.4 & 7.9 & 6.4 & 9.3 & 0.56 \\
sepsis (\%) & & & & & \\
\hline The & & & & \\
\hline
\end{tabular}

The $\chi^{2}$ test was used for statistical comparison, if not otherwise indicated ('Fisher's exact test). Bold text indicates significant associations.

B/C, blood culture.

We also performed a multivariable regression analysis in ethnically different subgroups (Caucasian, Turkey/Middle East). The FUT2 genotype had no effect on outcomes in the Caucasian population. In the much smaller subgroup of infants from Turkey/Middle East, A allele carriers were noted to have a potentially decreased risk of death during the primary stay in hospital (eight cases, 232 controls; OR: 0.21; 95\% CI: 0.05-0.8, $P=0.026$, data not shown).

\section{Treatment Strategies}

With regard to antibiotic treatment, infants with AA genotype were more often treated with cefotaxime (39.9 vs. $32.6 \%$; $P=0.003)$ and tobramycin (16.1 vs. $11.8 \%, P=0.01)$ as compared to GG/AG individuals, but no differences were observed for total use of antibiotics and administration of third line antibiotics such as carbapenems. In line with that, the FUT2 genotype did not influence the need for vasoactive drugs, diuretics, analgetics, inhalative drugs, surfactant, dexamethasone, insuline, or blood products. FUT2 genotype also had no impact on the need for mechanical ventilation, duration oxygen supplement, time until full enteral feeds $(150 \mathrm{ml} / \mathrm{kg})$, and duration of primary stay in hospital (data not shown).
Table 3. Pathogenic spectrum in blood-culture proven sepsis episodes

\begin{tabular}{lccc}
\hline $\begin{array}{l}\text { Pathogen in blood culture } \\
\text { (number of episodes, }(\%))\end{array}$ & $\begin{array}{c}\text { GG or GA } \\
n=1,655\end{array}$ & AA $n=386$ & $P$ \\
\hline CoNS & $126(7.7)$ & $32(8.3)$ & 0.5 \\
Group B streptococci & $6(0.4)$ & $1(0.3)$ & 0.8 \\
Staphylococcus aureus & $42(2.5)$ & $6(1.6)$ & 0.3 \\
Enterococcus species & $19(1.1)$ & $4(1)$ & 0.9 \\
Other streptococci & $1(0.1)$ & $1(0.3)$ & 0.3 \\
Pneumococcus & 0 & 0 & - \\
Escherichia coli & $15(0.9)$ & $3(0.8)$ & 0.8 \\
Klebsiella species & $23(1.4)$ & $3(0.8)$ & 0.3 \\
Enterobacter cloacae & $10(0.6)$ & $4(1)$ & 0.4 \\
Serratia & $1(0.1)$ & 0 & 0.6 \\
Proteus mirabilis & 0 & 0 & - \\
Pseudomonas aeruginosa & $3(0.2)$ & $1(0.3)$ & 0.8 \\
Listeria monocytogenes & $1(0.1)$ & 0 & 0.2 \\
Candida & $11(0.7)$ & $2(0.5)$ & 0.8 \\
ESBL E. coli & $2(0.1)$ & $1(0.3)$ & 0.5 \\
Others & $31(1.9)$ & $13(3.4)$ & 0.07 \\
\hline The Pvalues were derived from Fisher's Exact test. & &
\end{tabular}

Infection-Related Morbidities in the First Year of Life

As part of our follow-up, we sent questionnaires to parents of German Neonatal Network infants and compared subjectively reported infection-related morbidities in the first year of life with the data of a representative cross-sectional German health survey (KIGGS) (9). 1,411/2,406 questionnaires were answered for infection-related items. We found no genotyperelated differences for incidence of common cold, herpes-virus infection, bronchitis, croup, gastroenteritis/diarrhea, urinary tract infection, purulent conjunctivitis, and oral/diaper thrush. Parents noted that AA individuals were more often diagnosed with throat infection/tonsillitis (GG: $0.8 \%, n=3$, AG: $1.1 \%$, $n=6$; AA: $4.5 \%, n=9$; GG/AG vs. AA; $P<0.001$; fisher's exact test).

\section{DISCUSSION}

In a large well-characterized cohort of VLBW infants, we investigated the influence of the FUT2 $428 \mathrm{~A} / \mathrm{G}$ polymorphism on outcome measures. In contrast to previously published data (6), the FUT 2 genotype was not predictive for infections and other adverse outcomes.

The G428A polymorphism in the FUT2 gene is, in the Caucasian population, determining the so called secretor status. The FUT2 gene encodes for a $\alpha 1,2$-fucosyltransferase which is responsible for expressing $\mathrm{ABH}$ blood group antigens on mucosal surfaces and secretion of these antigens in body fluids. The polymorphism confers a nonsense mutation which results in a stop codon and subsequently leads to a nonfunctioning protein. The distribution of the polymorphism in our cohort is in concordance with existing literature $(7,8)$. Mothers of $6.2 \%$ of enrolled infants had Asian, African, or unknown ethnicity and the infants were excluded from analysis. In other populations, different 
Table 4. FUT 2 effect on neonatal outcomes adjusted for well-known clinical risk factors (multivariable regression analysis; very-low-birth weight infants $<32$ wk with Caucasian background)

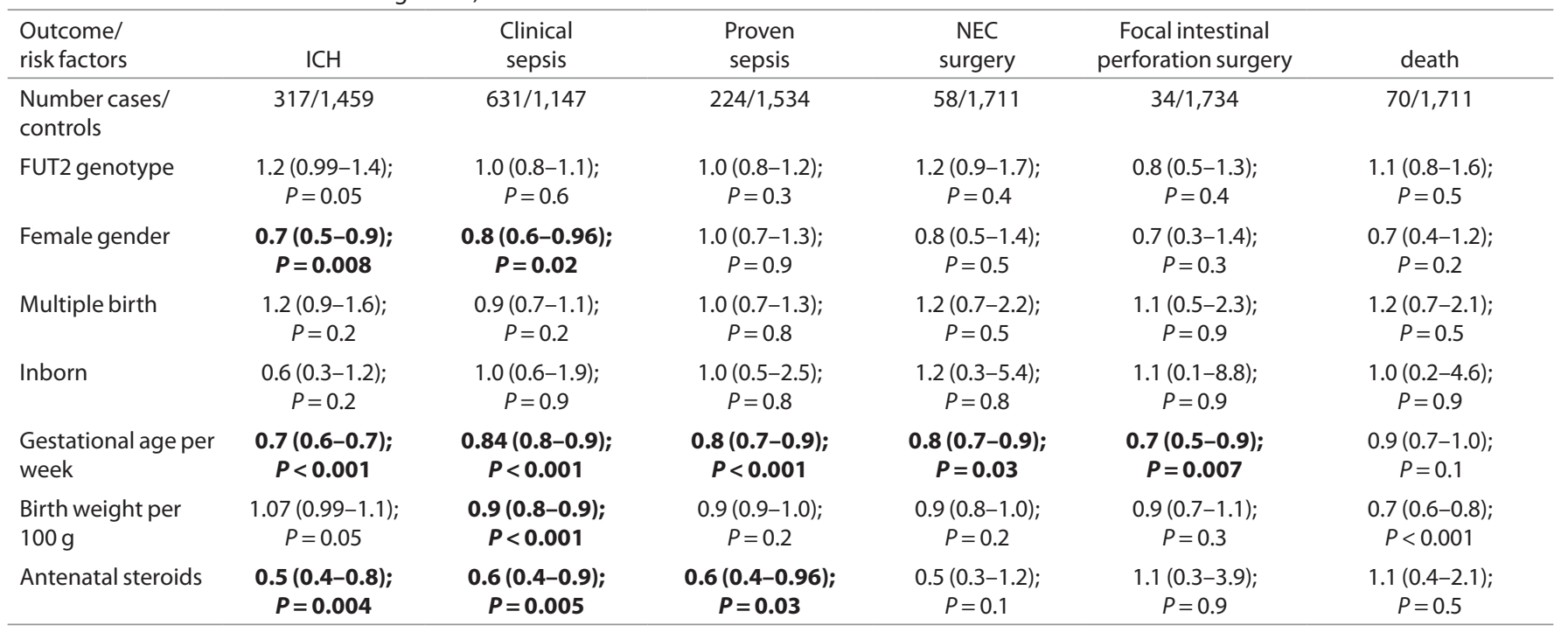

Effect sizes are given as odds ratios ( $95 \%$ confidence interval) for each outcome; bold text indicates significant associations.

$\mathrm{ICH}$, intracerebral hemorrhage; NEC, necrotizing enterocolitis.

polymorphisms (e.g., C571T) prevail within the FUT2 gene and determine secretor status.

Many data were published in recent years focusing on the secretor status and its association with mainly infectious and even other inflammatory diseases. Morrow et al. (6) were first to publish data on secretor status in VLBW infants and demonstrated an association between secretor genotype and death, Gram-negative sepsis, and NEC. This study included 410 preterm infants $<32$ gestational age. Given that death occurred in $13 \%$ of AA individuals ( $n=12$ out of 95) and 4\% in GG/AG individuals ( $n=13$ out of 299) in the Morrow study (6), our study had a statistical power of $>99 \%$ to detect the estimated difference (469 AA and 1,937 GG/AG individuals). In our study, none of the primary outcome measures proved to be associated with FUT2 genotype. In the first set of tests (GG/AG vs. AA), we assumed a recessive genetic model where two copies of the $\mathrm{A}$ allele are required to have an impact on the outcome of interest. We observed a tendency toward higher rates of early onset sepsis in AA individuals which was in part attributable to a combined effect of FUT 2 and PPROM. This observation underlines that future studies are needed to address potential interactions between maternal and infant genotypes related to adverse pregnancy outcomes, particularly preterm delivery, and PPROM. AA individuals were also noted to have a higher incidence of ICH. In a second set of tests, we assumed an additive genetic model and performed regression analysis in the subgroup of infants $<32 \mathrm{wk}$ of gestation in order to avoid over-representation of small-for gestational age infants and to improve comparability to Morrow's data. We found no specific genetic effect of FUT2 on outcomes of interest apart from a protective value of the A allele against death in the subgroup of infants with Turkish/Middle Eastern background. This subgroup, however, is very small and our data do not warrant a robust conclusion. In comparison with our setting, the study by Morrow et al. (6) was a single-center trial supported by functional data on the secretor status. Notably, secretor genotype and phenotype determined by salivary $\mathrm{H}$-antigen production are not always concordant. Low salivary $\mathrm{H}$ antigen production was observed among many heterozygous (GA) and some homozygous secretor (GG) individuals. Strengths of our present study are that it includes a large cohort of infants from multiple centers and includes well-defined in-hospital and follow-up clinical data obtained prospectively in a systematic manner. The major weakness is failure to measure the functional expression of the FUT2 gene ( $\mathrm{H}$-antigen). There are differences in population genetics, culture, and environment that clearly can account for the differences observed between this multicenter study and the previous study (6). We finally conclude that FUT2 genotype is not a good independent predictor of adverse infectious and inflammatory outcomes complicating prematurity.

With regard to infection-related morbidities in the first year of life, parents $(1,411 / 2,406$ questionnaires) noted that infants with AA genotype were more often diagnosed with throat infection/tonsillitis. This observation is interesting, in particular with respect to previous studies on the link of FUT2 and respiratory illness $(2,4,5)$, but this hypothesis needs to be evaluated in a prospective trial with larger cohorts.

Future studies regarding the secretor status and potential link with disease in preterm infants need to involve microbiota investigations, as previous reports demonstrated the link of mucosa-associated microbiota with FUT2 genotype $(10,11)$. It would also be interesting to study the interaction of fucoslytransferase activity with different aspects of the immune system in preterm infants, e.g., the regulation of assembly of the pre-B cell receptor and intracellular signalling for B-cell maturation (12).

In conclusion, we performed a large-scale study which did not confirm previous data on FUT2 genotype and infectious morbidity in preterm infants. It is important to acknowledge 
known clinical risk factors for infection in this vulnerable cohort of infants, i.e., gestational age and birth weight (13), in particular for future genome-wide association studies (14).

\section{METHODS}

\section{Genetic Association Study}

We prospectively studied the influence of the FUT-2 G428A polymorphism on several outcomes in 2,406 VLBW infants enrolled in a multicenter trial involving 47 neonatal intensive care units in Germany from March 2009 until December 2011 (German Neonatal Network). The inclusion criteria were as follows: birth weight $<1,500 \mathrm{~g}$ and gestational age $\leq 36+6 \mathrm{wk}$, exclusion criteria: lethal malformations, e.g., trisomy 13 and trisomy 18). After written informed consent was given by the parents, a DNA-sample of the infant was obtained by buccal swab and/or cord tissue and transferred to the study center (University of Lübeck). Antenatal and postnatal treatment and outcome data were recorded by according data sheets at the participating centers. After discharge, data sheets were sent to the study centre. A physician trained in neonatology evaluated the data quality by annual on site monitoring of completed data sets every 6 mo.

DNA was extracted using a commercial DNA purification kit (Quiagen, Hilden, Germany). The DNA was washed twice and eluted. Secretor genotype was determined at a single null mutation FUT2 G428A, Trp149 ->STOP (rs601338) by the TaqMan 5' nuclease assay (Applied Biosystems, Foster City, CA) and the 7900HT Fast RealTime PCR System.

\section{Definition of Outcome Measures}

Clinical sepsis was defined as sepsis with at least two clinical signs (15); i.e., temperature $>38{ }^{\circ} \mathrm{C}$ or $<36.5^{\circ} \mathrm{C}$, tachycardia $>200 / \mathrm{min}$, new onset or increased frequency of bradycardias or apneas, hyperglycemia $>140 \mathrm{mg} / \mathrm{dl}$, base excess $<-10 \mathrm{mval} / \mathrm{l}$, changed skin color, increased oxygen requirements) as well as antimicrobial therapy for at least $5 \mathrm{~d}$, no proof of causative agent in blood culture, no obvious site of infection, and one laboratory sign (C-reactive protein $>2 \mathrm{mg}$ / $\mathrm{dl}$, immature/neutrophil ratio $>0.2$, white blood cell count $<5 / \mathrm{nl}$, platelet count $<100 / \mathrm{nl})$.

Blood culture confirmed sepsis was defined by at least two clinical signs and one laboratory sign as mentioned above plus confirmation of causative agent in blood or cerebrospinal fluid (15).

All-cause mortality was defined as death occurring after admission to Neonatal Intensive Care Unit before discharge home. Bronchopulmonary dysplasia was diagnosed when needing oxygen or assisted ventilation evaluated at $36 \mathrm{wk}$ of postmenstrual age. ICH grades I-IV were diagnosed according to the ultrasound criteria of Papile (16). Cystic periventricular leukomalacia was defined as periventricular lesions. Clinical higher stage NEC was definied as NEC with Bell stage II/III. A further outcome parameter was surgery required for NEC or focal intestinal perforation (17). Higher stage retinopathy of prematurity was noted when treatment was required (18).

\section{Statistical Analysis}

Data analysis was performed using the SPSS 20.0 data analysis package (IBM, Munich, Germany). Hypotheses were evaluated with $\chi^{2}$ test, Fisher's exact test and Mann-Whitney $U$-test. In the first set of tests (GG/AG vs. AA), we assumed a recessive genetic model where two copies of the A allele are required to have an impact on the outcome of interest. A $P$ value $<0.05$ was considered as statistically significant for single tests.

In the second set of tests, we assumed an additive genetic model which was investigated in a multivariable logistic regression analysis. In order to allow for adjustment of more than one clinically important covariate, we included gestational age per week, birth weight per 100g steps, gender, multiple birth, inborn delivery, and exposure to antenatal steroids in order to describe the overall impact of the FUT2 genetic variant on the risk of the outcomes studied. Effect sizes were given as odds ratios and $95 \%$ confidence interval. The regression analysis was performed for VLBW infants $<32 \mathrm{wk}$ with full datasets. In addition, we performed regression analyses for subgroups of VLBW infants based on maternal ethnicity.

\section{Ethics}

The study parts were approved by the local committee on research in human subjects of the University of Lübeck and the local ethical committees at the other study centers.

\section{ACKNOWLEDGMENTS}

We are indebt to all doctors and nurses supporting this study by sample and data collection and to all parents and infants participating the study. We thank Michael Preuß and Andreas Ziegler for statistical review.

\section{STATEMENT OF FINANCIAL SUPPORT}

This study was funded by the Federal German Ministry of Education and Research (BMBF 01ER0805).

\section{Disclosure: None.}

\section{REFERENCES}

1. Thorven M, Grahn A, Hedlund KO, et al. A homozygous nonsense mutation $(428 \mathrm{G}->\mathrm{A})$ in the human secretor (FUT2) gene provides resistance to symptomatic norovirus (GGII) infections. J Virol 2005;79:15351-5.

2. Raza MW, Blackwell CC, Molyneaux P, et al. Association between secretor status and respiratory viral illness. BMJ 1991;303:815-8.

3. Carlsson B, Kindberg E, Buesa J, et al. The G428A nonsense mutation in FUT2 provides strong but not absolute protection against symptomatic GII.4 Norovirus infection. PLoS One 2009;4:e5593.

4. Innes $\mathrm{AL}, \mathrm{McGrath} \mathrm{KW}$, Dougherty $\mathrm{RH}$, et al. The $\mathrm{H}$ antigen at epithelial surfaces is associated with susceptibility to asthma exacerbation. Am J Respir Crit Care Med 2011;183:189-94.

5. Ronchetti F, Villa MP, Ronchetti R, et al. ABO/Secretor genetic complex and susceptibility to asthma in childhood. Eur Respir J 2001;17:1236-8.

6. Morrow AL, Meinzen-Derr J, Huang P, et al. Fucosyltransferase 2 nonsecretor and low secretor status predicts severe outcomes in premature infants. J Pediatr 2011;158:745-51.

7. McGovern DP, Jones MR, Taylor KD, et al.; International IBD Genetics Consortium. Fucosyltransferase 2 (FUT2) non-secretor status is associated with Crohn's disease. Hum Mol Genet 2010;19:3468-76.

8. Folseraas T, Melum E, Rausch P, et al. Extended analysis of a genome-wide association study in primary sclerosing cholangitis detects multiple novel risk loci. J Hepatol 2012;57:366-75.

9. Bergmann KE, Bergmann RL, Ellert U, Dudenhausen JW. [Perinatal risk factors for long-term health. Results of the German Health Interview and Examination Survey for Children and Adolescents (KiGGS)]. Bundesgesundheitsblatt Gesundheitsforschung Gesundheitsschutz 2007;50:670-6.

10. Rausch P, Rehman A, Künzel S, et al. Colonic mucosa-associated microbiota is influenced by an interaction of Crohn disease and FUT2 (Secretor) genotype. Proc Natl Acad Sci USA 2011;108:19030-5.

11. Wacklin P, Mäkivuokko $H$, Alakulppi N, et al. Secretor genotype (FUT2 gene) is strongly associated with the composition of Bifidobacteria in the human intestine. PLoS One 2011;6:e20113.

12. Li W, Liu Q, Pang Y, et al. Core fucosylation of $\mu$ heavy chains regulates assembly and intracellular signaling of precursor B cell receptors. J Biol Chem 2012;287:2500-8.

13. Tröger B, Göpel W, Faust K, et al.; German Neonatal Network. Risk for late-onset blood-culture proven sepsis in very-low-birth weight infants born small for gestational age: a large multicenter study from the German Neonatal Network. Pediatr Infect Dis J 2014;33:238-43.

14. Strunk T, Jamieson SE, Burgner D. Genetic and epigenetic susceptibility to early life infection. Curr Opin Infect Dis 2013;26:241-7.

15. Geffers C, Baerwolff S, Schwab F, Gastmeier P. Incidence of healthcareassociated infections in high-risk neonates: results from the German surveillance system for very-low-birthweight infants. J Hosp Infect 2008;68:214-21.

16. Papile LA, Burstein J, Burstein R, Koffler H. Incidence and evolution of subependymal and intraventricular hemorrhage: a study of infants with birth weights less than 1,500 gm. J Pediatr 1978;92:529-34.

17. Walsh MC, Kliegman RM. Necrotizing enterocolitis: treatment based on staging criteria. Pediatr Clin North Am 1986;33:179-201.

18. Garner A. The pathogenesis of ocular vascular disorders. Aust J Ophthalmol 1984;12:401-4. 